\title{
OBTENÇÃO DE EXTRATOS DE PIMENTA ROSA (Schinus terebinthifolius R.) UTILIZANDO PROPANO PRESSURIZADO E SOLVENTES ORGÂNICOS
}

\author{
K. S. ANDRADE ${ }^{1}$, M. V. TRES ${ }^{2}$, J. V de OLIVEIRA ${ }^{1}$, S. R. S. FERREIRA ${ }^{1}$ \\ ${ }^{1}$ Departamento de Engenharia Química e Alimentos \\ Universidade Federal de Santa Catarina \\ CEP 88040-900, Florianópolis, SC, Brasil \\ E-mail: sandra@enq.ufsc.br \\ ${ }^{2}$ Departamento de Engenharia de Alimentos, \\ Universidade Regional Integrada - URI, Campus de Erechim, \\ CEP 99700-000, Erechim - RS, Brasil
}

\begin{abstract}
RESUMO: Schinus terebinthifolius R. é uma árvore perene nativa da costa do Brasil. Muitas propriedades medicinais têm sido atribuídas a esta planta, como atividade antioxidante, cicatrizante e antitumoral. A extração por fluido pressurizado tem se mostrado uma alternativa aos processos convencionais de extração. Neste trabalho empregou-se extração com propano pressurizado, em pressões de 40, 50 e 60 bar, numa faixa de temperatura entre 35 e $55^{\circ} \mathrm{C}$. Os extratos obtidos foram comparados em termos de rendimento e atividade antioxidante àqueles obtidos em Soxhlet e ultrassom com etanol. $\mathrm{O}$ melhor rendimento das extrações com propano pressurizado foi obtido na pressão de 60 bar e $55^{\circ} \mathrm{C}$, sendo de 10 , $8 \%$, e percentual de atividade antioxidante pelo método DPPH inferior a $50 \%$. A extração em Soxhlet apresentou rendimento de 43, $7 \pm 0,9$, superior ao obtido com ultrassom, e atividade antioxidante de $95 \%$ pelo método DPPH.
\end{abstract}

\section{INTRODUÇÃO}

Schinus terebinthifolius Raddi (aroeira-pimenteira) é uma árvore perene nativa da costa do Brasil e que foi introduzida em outras regiões da América do Sul, América Central, Europa, Ásia e África. É conhecida por uma variedade de nomes como aroeira-vermelha, aroeira-pimenteira, pimenta brasileira, pimenta rosa, poivre rose, entre outros. A pimenta rosa tem sido utilizada em substituição a pimenta-do-reino e, segundo análises químicas relatadas na literatura, existe grande similaridade entre seus componentes químicos (BARBOSA; DEMUNER; CLEMENTE, 2007; LACA-BUENDIA et al, 1992).

Muitas propriedades medicinais têm sido atribuídas a esta planta, como atividade antioxidante, cicatrizante, antitumoral e antimicrobiana. No Brasil ela é utilizada na medicina popular como um anti-inflamatório e cicatrizante, além de possuir qualidades anti-nevrálgicas, adstringentes, tônicas e estimulantes (AMORIM; SANTOS, 2003; BERTOLDI, 2006).

A análise fitoquímica da pimenta rosa revelou a presença de taninos, alcalóides, flavonóides, saponinas esteroidais, esteróis, terpenos e uma grande quantidade de óleo essencial. 
Os óleos essenciais presentes nas folhas, frutos e casca são uma fonte importante de substâncias químicas. As folhas podem conter $1 \%$ de óleo essencial, enquanto que nos frutos esse valor pode chegar a 5\% (CARVALHO et al., 2003).

Os antioxidantes são substâncias capazes de inibir ou retardar significativamente a oxidação de substratos oxidáveis. A importância dos antioxidantes para os alimentos e sistemas biológicos é bem conhecida, uma vez que protegem os mesmos dos processos oxidativos (BISCAIA, 2007). O efeito do antioxidante consiste na inativação dos radicais livres, na complexação de íons metálicos ou na redução dos hidroperóxidos (ARAÚJO, 2004).

A técnica de extração empregada na obtenção de extratos de produtos naturais influencia diretamente a composição final dos extratos e consequentemente a sua qualidade. $O$ procedimento de extração é determinado pela família de compostos a ser extraída e também pelo objetivo do processo, se é quantitativo ou qualitativo. Ou seja, o rendimento de processo e a composição dos extratos dependem tanto do solvente utilizado como do método de extração aplicado.

Técnicas convencionais de extração com uso de solventes orgânicos, como ultrassom e Soxhlet, são comumente aplicadas nas indústrias química, farmacêutica e alimentícia na obtenção de variados extratos e podem utilizar uma ampla gama de solventes como metanol, hexano, clorofórmio, acetato de etila, acetona, éter, etc. No entanto, essas técnicas requerem um alto custo energético e podem degradar substâncias termicamente sensíveis, pois podem utilizar altas temperaturas de extração ou de separação da mistura soluto-solvente, dependendo da temperatura de ebulição do solvente (BISCAIA, 2007; CAMPOS, 2005; DÍAZ-REINOSO et al., 2006).

A extração com propano comprimido tem sido utilizada para obtenção de produtos naturais, com resultados interessantes (HEGEL et al., 2007; HAMDAN et al., 2008; FREITAS et al., 2008). Estudos têm mostrado que, em alguns casos, esse solvente é melhor para extração de óleos e produtos naturais quando comparado ao dióxido de carbono, que o solvente mais popular utilizado na tecnologia supercrítica. O propano é relativamente barato, não deixa resíduo tóxico e tem um alto poder de solvatação (SPARKS et al., 2006).

As pressões empregadas na obtenção de extratos utilizando propano são mais amenas do que aquelas usadas na extração com $\mathrm{CO}_{2}$ supercrítico. Economicamente, os processos com baixas pressões e temperaturas, podem reduzir custos na extração, com alto rendimento, em menor tempo, e consequentemente menor consumo de solvente, sendo possível, portanto, otimizar o processo (MESOMO et al., 2012).

O objetivo deste trabalho foi avaliar técnicas convencionais de extração e a extração com propano comprimido, comparando os resultados em termos de rendimento de extração e atividade antioxidante dos extratos de pimenta rosa.

\section{MATERIAIS E MÉTODOS}

\subsection{Matéria-prima e preparação da amostra}

As amostras de pimenta rosa (Schinus terebinthifolius R.) foram coletadas no campus da Universidade Federal de Santa Catarina. Após a colheita, realizou-se a limpeza dos grãos, removendo folhas, restos de galhos, poeira e demais sujidades presentes. As amostras foram 
submetidas à secagem em estufa com circulação de ar (De Leo, Porto Alegre/RS, Brasil) por 6 horas a $45^{\circ} \mathrm{C}$. Após a secagem, as pimentas foram trituradas em liquidificador doméstico e, em seguida, acondicionadas em embalagens de polietileno transparente e estocadas em freezer doméstico, a $-18^{\circ} \mathrm{C}$, até a realização dos experimentos.

\subsection{Extração com propano comprimido}

Os ensaios de extração foram realizados no Laboratório de Processos de Separação e Mistura/Termodinâmica na URI Erechim. Em cada ensaio de extração empregou-se $30 \mathrm{~g}$ de amostra seca e moída, e a vazão de solvente utilizada foi de $5 \mathrm{~mL} / \mathrm{min}$. O experimento inicia com o carregamento do extrator com propano e assim que os indicadores analógicos estão na pressão desejada, permiti-se a passagem do solvente pela coluna de extração, por meio da abertura da válvula de saída. Depois de finalizado este procedimento, a unidade extratora é conectada a um erlenmeyer, ao qual é acoplado internamente um tubo de ensaio para fazer a coleta do óleo, iniciando o processo de extração. Após pré-estabelecido o tempo de extração, fecha-se a válvula de entrada da coluna para que não haja mais a passagem de solvente pelo extrator, inicia-se a despressurização do sistema, retira-se o frasco contendo a amostra levando-o até uma capela para a saída completa do gás. Os ensaios foram conduzidos nas pressões de 40, 50 e 60 bar, e nas temperaturas de 35,45 e $55^{\circ} \mathrm{C}$, por 60 min cada um.

\subsection{Extrações a baixa pressão}

Os métodos de extração a baixa pressão utilizados nesse estudo foram extração em Soxhlet e extração assistida por ultrassom, que foram aplicadas empregando etanol como solvente.

A extração em Soxhlet foi realizada segundo a metodologia 920.39C da A.O.A.C. O procedimento consiste na recirculação de $150 \mathrm{~mL}$ de solvente sobre $5 \mathrm{~g}$ de amostra seca, em um aparato Soxhlet, durante $6 \mathrm{~h}$ de extração.

O método de extração por ultrassom foi conduzido conforme descrição de Jacques (2005) e Vinatoru (2001), no qual 7 gramas de amostra foram adicionados a $210 \mathrm{~mL}$ de solvente, acondicionados em balão de $250 \mathrm{~mL}$. O balão foi submetido a banho indireto em ultrassom utilizando-se uma lavadora ultrassônica, por 45 minutos.

Os extratos obtidos em cada método de extração foram submetidos a um processo de eliminação do solvente, em evaporador rotativo. As temperaturas de evaporação foram ajustadas para um nível abaixo do ponto de ebulição do solvente, a fim de evitar degradação térmica dos extratos. $\mathrm{O}$ vácuo foi ajustado a $650 \mathrm{mmHg}$.

O rendimento global $\left(\mathrm{X}_{0}\right)$ para todos os métodos de extração foi obtido a partir do valor médio dos experimentos, realizados em duplicata, considerando o razão entre a massa do extrato e da massa de matéria-prima utilizada.

\subsection{Atividade antioxidante pelo método DPPH}

A técnica de determinação de atividade antioxidante pelo método DPPH constitui na reação do composto em análise em uma solução etanólica de DPPH na concentração de $0,3 \mathrm{mM}$ e com concentrações crescentes $(5,10,25,50,125,250$ e $500 \mathrm{mg} / \mathrm{mL})$ dos extratos analisados, por 30 minutos, em temperatura ambiente e ao abrigo da luz. Decorrido o tempo de reação, faz-se a 
leitura das absorbâncias em espectrofotômetro (FEMTO, 800 XI, São Paulo, SP). Quanto maior a atividade antioxidante da amostra testada, mais estável o radical DPPH se torna, provocando a descoloração da solução (DPPH e extrato) e diminuindo sua absorbância (MENSOR et al, 2001). Assim, o percentual de inibição das amostras testadas sobre o radical pode ser calculado convertendo-se a absorbância em porcentual de atividade antioxidante (AA \%).

\section{RESULTADOS E DISCUSSÕES}

\subsection{Rendimento global de extração $\left(X_{0}\right)$}

Os resultados de rendimento obtidos para os diferentes métodos de extração empregados são apresentados na Tabela 1.

Tabela 1 - Rendimento global das extrações com propano pressurizado e etanol

\begin{tabular}{cccc}
\hline Método & Temperatura $\left({ }^{\circ} \mathbf{C}\right)$ & Pressão (bar) & $\mathbf{X}_{\mathbf{0}}(\boldsymbol{\%})$ \\
\hline Propano comprimido & 35 & 40 & 9,60 \\
Propano comprimido & 55 & 40 & 9,34 \\
Propano comprimido & 45 & 50 & 9,95 \\
Propano comprimido & 35 & 60 & 9,81 \\
Propano comprimido & 55 & 60 & 10,78 \\
Soxhlet etanol & - & - & $44 \pm 1$ \\
Ultrassom etanol & - & - & $21 \pm 2$ \\
\hline
\end{tabular}

Observando os dados da Tabela 1 pode-se perceber que a pressão exerce um efeito positivo no rendimento da extração com propano. No entanto, as diferenças entre os rendimentos nas condições estudadas foram pequenas, fazendo parte do erro experimental. Assim, é possível dizer que entre as faixas de pressão e temperatura estudadas o efeito dos parâmetros de processo foi pequeno e não influenciou fortemente o rendimento da extração.

A extração em Soxhlet apresentou rendimento superior ao obtido no processo com ultrassom. A justificativa desse comportamento reside na maior temperatura de operação, no reciclo do solvente e nas interações entre o solvente e matriz vegetal, característicos da extração em Soxhlet, que podem contribuir para uma maior solubilização de compostos, de diferentes classes, elevando assim o rendimento da extração (Markon et al., 2007).

A diferença observada entre os resultados dos dois solventes (propano e etanol) foi justificada pela polaridade dos solventes empregados, sugerindo a predominância de compostos polares nos extratos de pimenta rosa, mais facilmente solubilizados pelo etanol, comparado com o propano.

\subsection{Atividade antioxidante}


Os resultados de atividade antioxidante de todos os extratos de pimenta rosa obtidos pelos diferentes métodos, determinados pelo método DPPH são apresentados na Tabela 2 e comparados com o antioxidante sintético BHT, utilizado como padrão para as análises.

Tabela 2 - Potencial antioxidante dos extratos de pimenta rosa avaliado pelo método DPPH

\begin{tabular}{|c|c|c|c|}
\hline EXTRAÇÃO & SOLVENTE & AA\% $(500 \mu \mathrm{g} / \mathrm{mL})$ & $\mathrm{EC}_{50}(\boldsymbol{\mu g} / \mathrm{mL})$ \\
\hline Ultrassom & $\mathrm{EtOH}$ & 66 & 339 \\
\hline Soxhlet & $\mathrm{EtOH}$ & 95,5 & 80 \\
\hline $\mathrm{PC} 40 \mathrm{bar} / 35^{\circ} \mathrm{C}$ & Propano & 40,8 & $>1000$ \\
\hline $\mathrm{PC} 40 \mathrm{bar} / 55^{\circ} \mathrm{C}$ & Propano & 3 & $>1000$ \\
\hline PC $50 \mathrm{bar} / 45^{\circ} \mathrm{C}$ & Propano & 3 & $>1000$ \\
\hline PC $60 \mathrm{bar} / 35^{\circ} \mathrm{C}$ & Propano & 2,5 & $>1000$ \\
\hline PC 60 bar $/ 55^{\circ} \mathrm{C}$ & Propano & 3,3 & $>1000$ \\
\hline BHT & - & 89,7 & 261 \\
\hline
\end{tabular}

PC: propano comprimido

Entre os extratos avaliados, aquele obtido em Soxhlet com etanol apresentou maior atividade antioxidante, superior ao valor determinado para o antioxidante padrão BHT. Neste caso, o valor de EC50 foi de $80 \mu \mathrm{g} / \mathrm{mL}$, o que caracteriza o forte potencial antioxidante da amostra. Segundo Campos et al. (2008), valores de EC50 abaixo de $250 \mu \mathrm{g} / \mathrm{mL}$ são considerados de alto potencial antioxidante.

Em relação aos extratos obtidos com propano comprimido, apenas a condição de 40 bar e $35^{\circ} \mathrm{C}$ apresentou atividade antioxidante superior a $40 \%$, ainda assim, este resultado é bastante contraditório, visto que o valor de EC50 foi superior a $1000 \mu \mathrm{g} / \mathrm{mL}$. O EC50 é calculado em função das atividades antioxidantes encontradas para cada concentração testada, e para este extrato em especial, em todas as concentrações, que variaram de 5 a $500 \mu \mathrm{g} / \mathrm{mL}$, a atividade antioxidante determinada foi aproximadamente $40 \%$.

\section{CONCLUSÃO}

O etanol mostrou-se um solvente adequado para a obtenção de extrato de pimenta rosa tanto em relação ao rendimento da extração, como em termos de potencial antioxidante dos extratos.

Por conta da recirculação de solvente que ocorre na extração em Soxhlet, esta metodologia apresentou o melhor rendimento de extração.

Embora a extração com propano comprimido tenha apresentado bons resultados de rendimento, os resultados obtidos pelo método DPPH sugerem que os extratos possuem baixo potencial antioxidante. No entanto, uma avaliação mais geral, incluindo outros métodos de avaliação de atividade antioxidante e ainda a verificação do perfil químico dos extratos faz-se necessária a fim de confirmar o potencial biológico dos extratos de pimenta rosa.

\section{REFERÊNCIAS BIBLIOGRÁFICAS}


AMORIM, M. M. R.; SANTOS, L. C. Tratamento da vaginose bacteriana com gel vaginal da aroeira (Schinus terebinthifolius Raddi): ensaio clinico randomizado. Revista Brasileira de Ginecologia e Obstetrícia, Rio de Janeiro, v. 25, n. 2, p. 95-102, 2003.

Association of Official Analytical Chemists (2005), Official Methods of Analysis of AOAC International, Gaithersburg, Maryland, USA.

ARAÚJO, J. M. A. Química de Alimentos: Teoria e Prática. 4.ed. Belo Horizonte. Editora UFV - Universidade Federal de Viçosa, 2004. 415p.

BARboSA, L. C. A.; DEMUNER, A. J.; CLEMENTE, A. D. Seasonal variation in the composition of volatile oils from Schinus terebinthifolius Raddi. Química Nova, v. 30, p. 1959-1965, 2007.

BERTOLDI, M. C. Atividade antioxidante in vitro da fração fenólica, das oleorresinas e do óleo essencial de pimenta rosa (Schinus terebinthifolius Raddi). 2006. Dissertacao (Mestre em Ciências e Tecnologia de Alimentos) - Universidade Federal de Vicosa, Vicosa, MG.

BISCAIA, D. Comparação entre tecnologia supercrítica e técnicas convencionais de extração para obtenção de extratos de própolis avaliados através de suas atividades biológicas. Florianópolis: UFSC, 2007. Dissertação (Mestrado), Departamento de Engenharia Química e Engenharia de Alimentos, Universidade Federal de Santa Catarina, 2007.

CAMPOS, L. M. A. S.; MICHIELIN, E. M. Z.; DANIELSKI, L.; FERREIRA, S. R. S. Experimental data and modeling the supercritical fluid extraction of marigold (Calendula officinalis) oleoresin. Journal of Supercritical Fluids, v. 34, p. 163-170, 2005.

CAMPOS, L. M. A. S.; LEIMANN, F. , V.; PEDROSA, R. C.; FERREIRA, S. R. S. Free radical scavenging of grape pomace extracts from Cabernet sauvingnon (Vitis vinifera). Bioresource Technology, v. 99, p. 8413-20, 2008.

CARVALHO, M. C. R. D., BARCA, F. N. T. V., AGNEZ-LIMA, L. F., MEDEIROS, S. R. B. Research article evaluation of mutagenic activity in extract of pepper tree skin bark (Schinus terebinthifolius Raddi). Environ. Mol. Mutagen. V. 42, p. 185 - 191, 2003.

DIAZ-REINOSO, B.; MOURE, A.; DOMINGUEZ, H.; PARAJO, J. C. Supercritical CO2 Extraction and Purification of Compounds with Antioxidant Activity. Journal of Agricultural and Food Chemistry, v. 54, n. 7, p. 2441-2469, 2006.

HAMDAN, S., DAOOD, H. G., MARKUS, M. T., ILLÉS, V. Extraction of cardamom oil by supercritical carbondioxide and sub-critical propane. J. of Supercritical Fluids, v. 44, p. 25-30, 2008. 
HEGEL, P. E.; ZABALOY, M. S.; MABE, G. D. B.; PEREDA, S.; BRIGNOLE, E. A. Phase equilibrium engineering of the extraction of oils from seeds using carbon dioxide + propane solvent mixtures. Journal of Supercritical Fluids, 42, 318-324, 2007.

JACQUES, R. S. Caracterização química da erva mate (Ilex paraguariensis): aplicação de diferentes processos de extração e influência das condições de plantio sobre a composição química. Porto Alegre: UFRGS, 2005. Tese (Doutorado em Química), Instituto de Química, Universidade Federal do Rio Grande do Sul, 2005.

LACA -BUENDIA, J. P.; BRANDÃO , M.; OLIVEIRA, L. M. da S. Utilização dos frutos de Schinus terebinthifolius Raddi. (Anacardiaceae) na substituição da pimenta-do-reino (Piper nigrum 1.). Daphne, Belo Horizonte, v. 2, n. 4, p. 34-36, jul., 1992.

MARKON, M.; HASAN, M.; DAUD, W. R.; SING, H.; JAHIM, J. M. Extraction of hydrolysable tannins from Phyllanthus niruri Linn.: Effects of solvents and extraction methods. Separation and Purification Technology, v. 52, p. 487-496, 2007.

MENSOR, L. L.; MENEZES, F. S.; LEITÃO, G. G.; REIS, A. S.; SANTOS, T. C.; COUBE, C. S.; LEITÃO, S. Screening of Brazilian plant extracts for antioxidant activity by the use of DPPH free radical method. Phytotherapy Research, v. 15, p. 127-130, 2001.

MESOMO, M. C.; SCHEER, A. DE P.; PEREZ, E.; NDIAYE, P. M.; CORAZZA, M. L. Ginger (Zingiber officinale R.) extracts obtained using supercritical $\mathrm{CO} 2$ and compressed propane: Kinetics and antioxidant activity evaluation. Journal of Supercritical Fluids, 71, 102-109, 2012.

SPARKS, D.; HERNANDEZ, R.; ZAPPI, M.; BLACKWELL, D.; FLEMING, T. Extraction of rice bran oil using supercritical carbon dioxide and propane. Journal of the American Oil Chemists' Society, 83, 885-891, 2006.

VINATORU, M. An overview of the ultrasonically assisted extraction of bioactive principles from herbs. Ultrasonics Sonochemistry, v. 8, p. 303-13, 2001. 